\title{
Reanimación hídrica: tipos de líquidos y efectos adversos
}

\section{Graciela Merinos-Sánchez ${ }^{1}$, Luis Antonio Gorordo-Delsol², John Oswaldo Guamán-Crespo ${ }^{1}$ y Guillermo David Hernández-López²}

${ }^{1}$ Servicio de Urgencias, Hospital General de México «Dr. Eduardo Liceaga»; ${ }^{2}$ Unidad de Cuidados Intensivos Adultos, Hospital Juárez de México. Ciudad de México, México

\section{Resumen}

La adecuada reanimación hídrica requiere de un profundo entendimiento de la fisiología de los fluidos, de los mecanismos de regulación del endotelio y de la composición de cada tipo de solución intravenosa que se utilice. Este trabajo aborda los puntos clave del desarrollo de las soluciones a través de la historia, contrasta los cánones fisiológicos y patológicos de la regulación hemodinámica y endotelial de los fluidos, y concluye describiendo la composición de diversas soluciones y grupos, con sus efectos benéficos y adversos, todo sobre la base de la evidencia más reciente de la literatura internacional.

Palabras clave: Terapia hídrica. Fenómeno fisiológico cardiovascular. Medicina de Urgencias. Medicina crítica.

\section{Fluid resuscitation: types of solutions and adverse effects}

\begin{abstract}
Adequate fluid resuscitation requires a deep understanding of the physiology of liquids, the mechanisms of regulation of the endothelium and the composition of each type of intravenous solution used. This work addresses the key points of the development of solutions throughout history, contrasts the physiological and pathological canons of hemodynamic and endothelial regulation of fluids, and concludes by describing the composition of various solutions, groups, beneficial and adverse effects, all on the basis of the most recent evidence from international literature.
\end{abstract}

Key words: Fluid therapy. Cardiovascular physiological phenomena. Emergency Medicine. Critical care.

\section{Correspondencia:}

*Luis Antonio Gorordo-Delsol

Av. Instituto Politécnico Nacional, 5160

Col. Magdalena de las Salinas

Del. Gustavo A. Madero

Ciudad de México, Mexico

E-mail: luis.gorordodelsol@icloud.com bajo la licencia CC BY-NC-ND (http://creativecommons.org/licenses/by-nc-nd/4.0/).
Fecha de recepción: 10/12/2018

Fecha de aceptación: 04/01/2019

DOI: 10.24875/REIE.M19000002
Disponible en internet: 08-02-2019 Rev Educ Investig Emer. 2019;1(1):23-31 www.medicinadeemergencias.com 


\section{Introducción}

La reanimación hídrica intravenosa tiene sus orígenes en 1831: mientras se combatía la epidemia de cólera en Irlanda, el Dr. William Brooke O'Shaughnessy notó que la sangre de los pacientes fallecidos por cólera se encontraba más "oscura y espesa", e hizo observaciones sobre el «estancamiento universal del sistema venoso y el rápido cese de la arterialización de la sangre»; además estudió la pérdida en las proporciones entre el agua y las sales de la sangre ${ }^{1-4}$. Un año después de la epidemia de cólera de 1831, Thomas Aitchison Latta logró infundir 12 I de solución a seis pacientes con cólera y describió sus resultados inmediatos sobre el pulso y la respiración: «tan inmediato y profundo, aparentemente capaz de reanimar muertos ${ }^{2}$. Cerca de 50 años después, Singer Ringer estudió y describió las propiedades de las diferentes sales en el protoplasma con soluciones a las que agregó calcio, sodio, cloro y potasio, pues consideró que el calcio y otros electrólitos podrían tener efectos cardiotónicos ${ }^{4}$. No fue hasta 1930 cuando la solución de Ringer fue modificada por Alexis Frank Hartmann, quien tomó la decisión de añadir lactato como elemento amortiguador ${ }^{3,4}$.

Durante el siglo XVI el Parlamento francés $-\mathrm{y}$, por consiguiente, gran parte de Europa- prohibió la transfusión sanguínea. Robertson, durante la Primera Guerra Mundial, comenzó a conservar sangre citrada y a transfundirla guiándose por los niveles de hemoglobina ${ }^{5,6}$. El Servicio de Transfusión de Barcelona reportó el uso de más de 9,000 I de sangre durante la Guerra Civil Española (1936-1939). El uso de plasma para reanimación se diseminó hasta su acmé en la guerra de Corea, se limitó por múltiples reportes de hepatitis $\mathrm{B}$ y se cambió por dextrán y albúmina, particularmente durante los ataques a Pearl Harbor en $1941^{6}$. En la década de 1970 William C. Shoemaker estableció la reanimación guiada por metas supranormales con apoyo de soluciones y fármacos ${ }^{7}$. Desde entonces, la polémica ha escalado con estudios en modelos animales y humanos buscando la solución ideal para diferentes enfermedades e incluso grados y etapas de una misma enfermedad ${ }^{8}$.

\section{Fisiología de los líquidos}

En 1966 Luft descubrió «la fina estructura capilar y la capa endocapilar" del sistema circulatorio y en 1980 Curry y Michel propusieron la teoría del tamizado molecular de la pared capilar, en la que se describe una matriz de fibras moleculares que cubre las células endoteliales en el lado luminal del capilar ${ }^{9}$. Esta estructura se conoce como glucocáliz y está constituida por glicoproteínas y proteoglicanos de la familia de los sindecanos y glipicanos que llevan glucosaminoglicanos lineales altamente sulfatados -principalmente heparán-sulfato, condroitín-sulfato y dermatán-sulfato- y ácido hialurónico, que se encuentra unido a receptores que forman una malla estrecha de carga eléctrica negativa $^{10}$. Constituye el $25 \%$ del espacio intravascular total, más delgado en la microcirculación $(0.2 \mathrm{~mm})$ y más grueso en los grandes vasos $(0.8 \mathrm{~mm})$, y representa un esqueleto basal que interactúa intensa y dinámicamente con todo tipo de elementos plasmáticos. El fluido dentro del glucocáliz constituye una porción no circulante del volumen intravascular que mantiene un gradiente de concentración de proteínas entre el plasma de flujo libre y las hendiduras intercelulares del endotelio ${ }^{10,11}$.

Las funciones del glucocáliz incluyen la regulación de la permeabilidad vascular y la limitación de la adhesión plaquetaria y leucocitaria al endotelio -al restringir el contacto de las moléculas plasmáticas con el endotelio-; asimismo, permite el acoplamiento de enzimas agonistas y antagonistas, favorece la regulación de la coagulación, es un amortiguador del cizallamiento $y$, junto con otras moléculas, regula la producción de sintasa de óxido nítrico, la vasodilatación y la perfusión selectiva ${ }^{10,11}$. Cuando se produce una lesión en el endotelio o la degeneración del glucocáliz, sobreviene una pérdida de glucosaminoglicanos -proceso llamado compactación o descamación- generada por situaciones como la infusión rápida de cristaloides, el choque séptico, la hiperglucemia o un trauma severo ${ }^{12-14}$. El daño es medible en la sangre con el aumento de glucosaminoglicanos como producto de desecho debido al desprendimiento o destrucción del glucocáliz, encontrando sindicano 1, heparán-sulfato, ácido hialurónico y condroitín-sulfato ${ }^{15-17}$.

Entender la función del glucocáliz permitió renovar la ecuación de Starling, donde la presión oncótica del líquido intersticial es sustituida por la presión del subglucocáliz -que se mantiene libre de proteínas con un gradiente cercano a cero- y comprender mejor el intercambio transvascular de los fluidos. Se establece que los capilares no fenestrados normalmente filtran fluido al espacio intersticial a lo largo de su recorrido y la absorción a través de los capilares venosos y las vénulas no ocurre, pues la presión oncótica en este punto -venosa- no invierte filtración desde el intersticio, sino que la mayor parte del fluido filtrado de las arterias hacia el intersticio retorna por la circulación como linfa9 


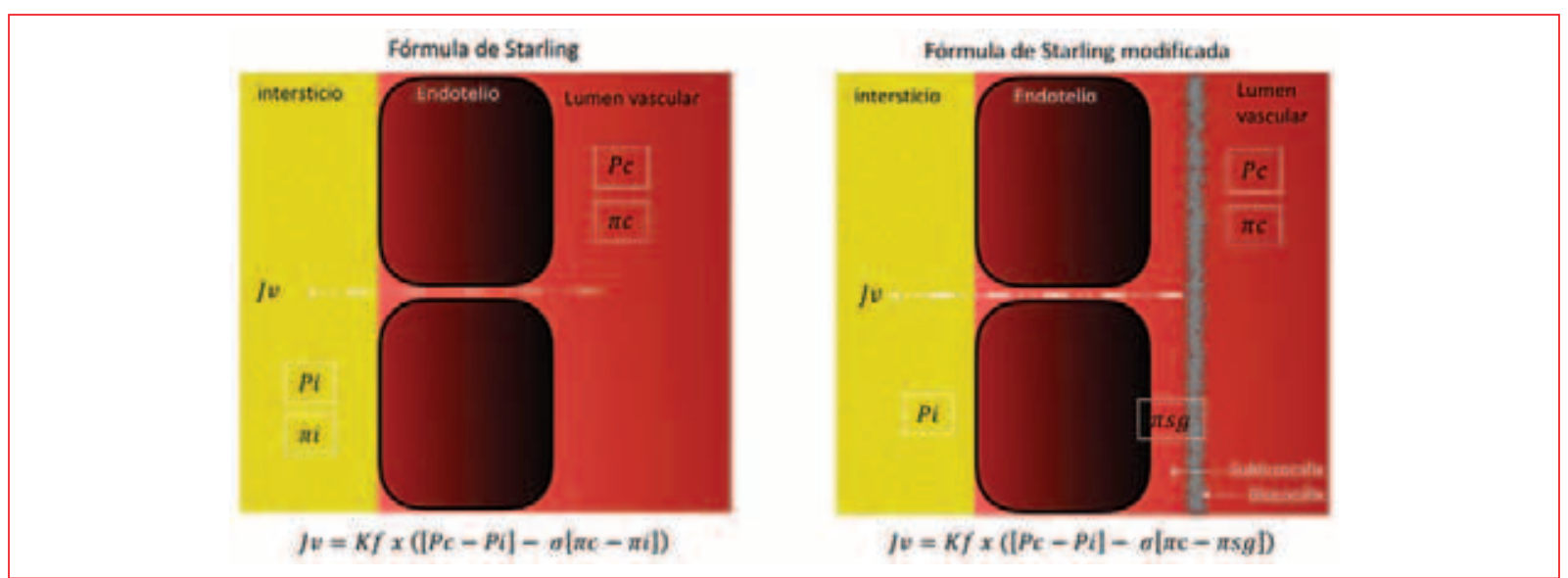

Figura 1. Esquemas del glucocáliz.

La fórmula de Starling clásica se expresa de la siguiente forma:

$$
\mathrm{JV}=\mathrm{Kf} \times([\mathrm{Pc}-\mathrm{Pi}]-\sigma[\pi \mathrm{c}-\pi \mathrm{i}])
$$

Donde Jv es la filtración transendotelial (o transcapilar) por segundo $\left(\mathrm{m}^{3} / \mathrm{s}\right)$; Kf, el producto de $\mathrm{Lp}$, que es la conductividad hidráulica de la membrana $(\mathrm{m} / \mathrm{s} /$ $\mathrm{mm} \mathrm{Hg}$ ) por $\mathrm{S}$, el área de la superficie de filtración $\left(\mathrm{m}^{2}\right)$; Pc, la presión hidrostática capilar; Pi, la presión hidrostática intersticial; $\sigma$, el coeficiente de reflexión de Staverman (grado en que se resiste una macromolécula a cruzar la barrera endotelial); $\pi$ c, la presión oncótica de las proteínas plasmáticas, y $\pi \mathrm{i}$, la presión oncótica intersticial.

Levick, et al. proponen que un sistema de pequeños poros transvasculares semipermeables debajo del glucocáliz es la verdadera capa que cubre las hendiduras intercelulares del endotelio y separa al plasma de una «región protegida», el espacio del subglucocáliz, que está casi libre de proteínas ${ }^{18}$, es decir, que el flujo transendotelial depende de las características de los capilares (fenestrados, no fenestrados o continuos), de la presión del flujo capilar y del gradiente generado por un espacio libre de proteínas (presión oncótica del subglucocáliz), y la presión oncótica de las proteínas plasmáticas -incluida la albúmina- escapa al espacio intersticial por un número escaso de poros grandes, que son responsables del aumento del Jv observado en las fases tempranas de la inflamación, y esto es susceptible de intervención farmacológica ${ }^{19}$. Finalmente el flujo transcapilar es mucho menor que el predicho por el principio de Starling y la linfa es la ruta mayor para retornar a la circulación. Sin embargo, elevar la presión oncótica del plasma reduce el flujo transcapilar, pero no causa absorción.
La fórmula de Starling según el modelo del glucocáliz se expresa de la siguiente forma:

$$
\mathrm{JV}=\mathrm{Kf} x([\mathrm{Pc}-\mathrm{Pi}]-\sigma[\pi \mathrm{c}-\pi \mathrm{sg}])
$$

Donde Jv es la filtración transendotelial neta por segundo $\left(\mathrm{m}^{3} / \mathrm{s}\right)$; Kf, el coeficiente de filtración -producto de Lp x S-; Pc, la presión hidrostática capilar; Pi, la presión hidrostática intersticial; $\sigma$, el coeficiente de reflexión de Staverman; $\pi c$, la presión oncótica de las proteínas plasmáticas, y $\pi \mathrm{sg}$, la presión oncótica del subglucocáliz (Fig. 1).

\section{Cristaloides}

Los cristaloides generalmente están constituidos por agua y sales minerales $u$ orgánicas que se diluyen y difunden junto con el disolvente -léase agua- cuando tiene que atravesar una membrana porosa ${ }^{20}$. Hartog Jacob Hamburger descubrió que los eritrocitos humanos sufrían menos lisis en una concentración de cloruro de sodio al $0.9 \%$, y así se estableció que una solución de estas características se consideraría «fisiológica» ${ }^{21}$. La mayoría de las soluciones cristaloides se distribuyen bien en el líquido intra y extracelular, con un alto índice de eliminación, y a los 60 min de su infusión sólo permanece el $20 \%$ del volumen infundido en el espacio intravascular22.

De acuerdo al principio de electroneutralidad del modelo de Stewart, el plasma tiene tres variables independientes para mantener el equilibrio: la presión parcial de oxígeno, la SID y los ácidos débiles no volátiles; las modificaciones en dichas variables condicionarán cambios en el pH. La SID en el plasma es de $40 \mathrm{mEq} / \mathrm{l}$, que corresponde a la resta de los cationes y los aniones $\left(\mathrm{Na}^{+}, \mathrm{K}^{+}, \mathrm{Ca}^{+}, \mathrm{Mg}^{+}, \mathrm{Cl}^{-}\right.$, lactato, cetoácidos, aniones 


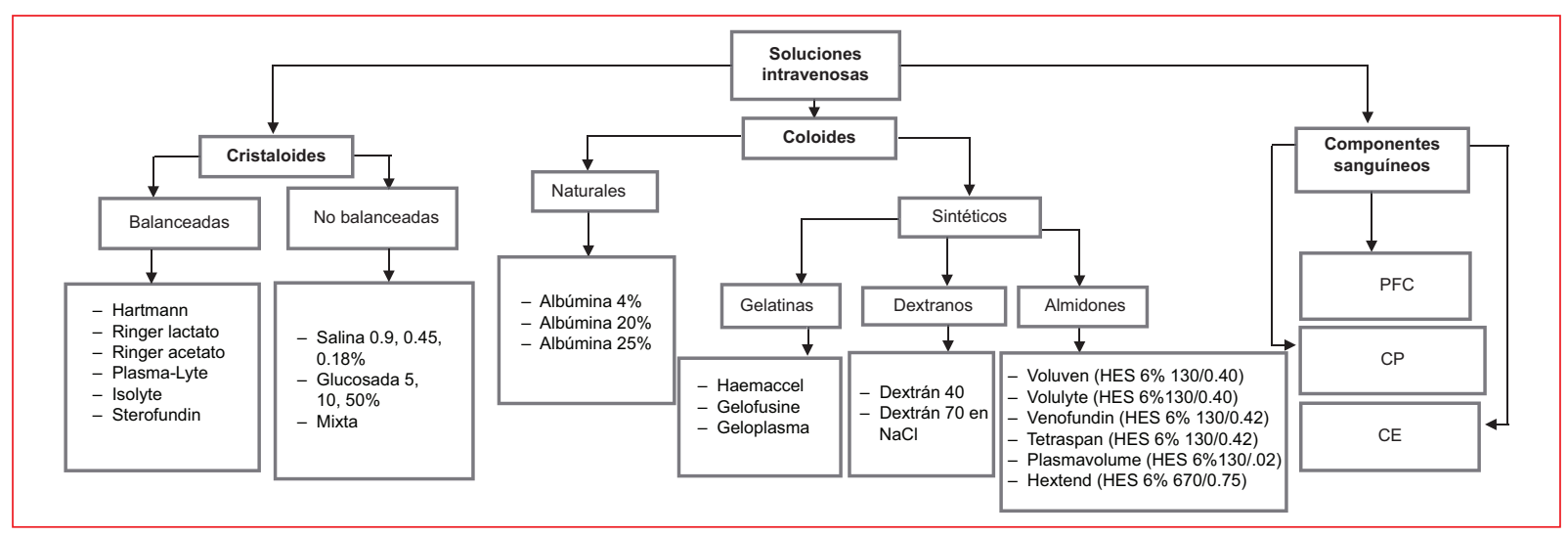

Figura 2. Tipos de soluciones.

PFC: plasma fresco congelado, CP: concentrado plaquetario, CE: concentrado eritrocitario.

orgánicos), mientras que la SID de la solución salina al $0.9 \%$ es de cero, es decir, sobrerreanimar a un paciente con solución salina al $0.9 \%$ genera una disminución de la SID del plasma creando tendencia a la acidosis -en este caso, acidosis hiperclorémica-, que se asocia con aumento de la mortalidad, disminución de flujos urinarios, coagulopatía, inflamación, disminución de la función miocárdica y respuesta a catecolaminas, y arritmias, entre otros ${ }^{23-25}$. La solución Hartmann, por ejemplo, tiene una SID de $28 \mathrm{mEq} / \mathrm{l}$; Plasma-Lyte, de 50 $\mathrm{mEq} / \mathrm{l}$, y Sterofundin, de $29 \mathrm{mEq} / \mathrm{l}$, que se considera más parecido al plasma, con menor contenido de cloro; por eso entran en el grupo de soluciones balanceadas (Fig. 2).

\section{Soluciones no balanceadas}

Las soluciones no balanceadas son todas aquéllas que tienen osmolaridad, tonicidad, contenido electrolítico y, por tanto, una brecha de iones fuertes (SID) distinta al plasma. La más utilizada de este grupo es la solución salina al $0.9 \%$, mal llamada «fisiológica», pues es hipertónica y ácida en relación con el plasma, y contiene una cantidad supranormal de sodio y cloro, con una SID de $0 \mathrm{mEq} / \mathrm{l}$. El mismo efecto en la SID tienen otras mezclas de $\mathrm{NaCl}$, por ejemplo la hipotónica («al medio») al $0.45 \%$, la hipertónica al $3 \%$ u otras concentraciones. También las soluciones glucosadas tienen una SID de $0 \mathrm{mEq} / \mathrm{I}$ con una osmolaridad de 277 $\mathrm{mEq} / \mathrm{l} \mathrm{al} 5 \%, 555 \mathrm{mOsm} / \mathrm{l} \mathrm{al} 10 \%$ y así sucesivamente hasta los 2,777 mOsm/l al $50 \%$, es decir, a mayor concentración de glucosa, mayor osmolaridad. Sin embargo, las soluciones glucosadas no contienen electrólitos, lo que confiere un coeficiente de reflexión de Staverman en extremo bajo, de ahí que se conozcan como «agua libre», pues su permanencia en el espacio intravascular es muy corta ${ }^{21,22,26}$.

Las soluciones no balanceadas tienen en común varios efectos adversos, todas hacen un efecto de dilución, mientras que las que contienen un alto contenido de $\mathrm{NaCl}$ generan hipercloremia y eventualmente acidosis. Los efectos adversos más relevantes son:

- Alteración de la contractibilidad del músculo esquelético y cardíaco por la disminución en la sensibilidad al calcio, disminución de la concentración intracelular de potasio y acidosis.

- Vasoconstricción renal, incremento de las resistencias vasculares renales y disminución de la filtración glomerular.

- Hay poca evidencia de alteraciones de la coagulación relacionadas con el tipo de líquido, y muchas están relacionadas con el volumen y balance hídrico positivo con compactación del glucocáliz y liberación de anticoagulantes, que alargan los tiempos de coagulación y favorecen las hemorragias 9,11,22,24,26,27.

- Las soluciones no balanceadas hipotónicas (solución salina al $0.18 \%$, solución glucosada) se han relacionado con hiponatremia en población pediátrica y a su vez incremento en la mortalidad ${ }^{28}$.

\section{Soluciones balanceadas}

Las soluciones balanceadas son aquéllas que tienen una SID de entre 24 y $29 \mathrm{mEq} / \mathrm{l}$, un amortiguador (lactato, acetato, malato) y cloro menor o igual a $110 \mathrm{mEq} / \mathrm{l}$-éstas también se llaman «bajas en cloro», en lugar de balanceadas-. La solución Hartmann, por ejemplo, tiene una SID de $28 \mathrm{mEq} / \mathrm{l}$, con variantes de acuerdo a su amortiguador (Ringer lactato, Ringer acetato); Plasma-Lyte 148 contiene una SID de $50 \mathrm{mEq} / \mathrm{l}$ con 
acetato y gluconato como amortiguador; y Sterofundin, $29 \mathrm{mEq} / \mathrm{l}$ más acetato y malato, lo cual genera menor desequilibrio ácido-base. Sin embargo, no todas las soluciones se encuentran en México ${ }^{26}$ (Tabla 1).

En la última década se han publicado decenas de estudios que comparan soluciones no balanceadas con balanceadas. En la tabla 2 se resumen algunos de los más importantes en relación con la reanimación hídrica ${ }^{29-37}$.

\section{Coloides}

Los coloides son suspensiones de partículas sólidas muy finas, de $10^{-9}$ a $10^{-5 m}$, que no se difunden con su disolvente cuando éste tiene que atravesar una membrana porosa ${ }^{20}$. Las macromoléculas pueden estar elaboradas a partir de proteínas o carbohidratos, por lo que todos los coloides disponibles en el mercado son sintéticos -excepto la albúmina-, lo que genera una gran variedad de productos. El gran peso molecular, teóricamente, confiere un alto coeficiente de reflexión de Staverman; sin embargo, en la práctica clínica el líquido en el que estas macromoléculas están suspendidas puede difundir pasivamente al intersticio o por medio de gradientes de presión oncótica ${ }^{1,9,19,22}$.

La albúmina es la proteína más abundante en el plasma humano (50-60\%); formada por una sola cadena polipeptídica de 585 aminoácidos y con un peso molecular de 66 a $69 \mathrm{kDa}$, aporta el $80 \%$ de la presión oncótica del plasma ${ }^{38}$. Para fines clínicos se divide en dos grupos: isooncótica (al $405 \%$ ) e hiperoncótica (al 20-25\%), y esto se debe tener en consideración en el momento de interpretar los estudios publicados, pues los efectos no son iguales. La Food and Drug Administration (FDA) de EE.UU. estableció como estándar que la albúmina humana al $5 \%$ se compone de proteínas (> $96 \%$ de albúmina humana) $=50 \mathrm{~g} / \mathrm{l}$, sodio $=130 \mathrm{a}$ $160 \mathrm{mmol} / \mathrm{l}$, potasio $<2 \mathrm{mmol} / \mathrm{l}, \mathrm{N}$-acetil-DL-triptófano = 0.064-0.096 $\mathrm{mmol} / \mathrm{g}$ de proteína y ácido caprílico = 0.064-0.096 mmol/g de proteína, mientras que la albúmina al $21 \%$ tiene $210 \mathrm{~g} / \mathrm{l}$ de proteínas y menos de 130 $\mathrm{mmol} / \mathrm{l}$ de sodio.

El metaanálisis de Vincent, et al. concluye que la albúmina disminuye el riesgo relativo (RR) de morbilidad (muerte o complicaciones cardiovasculares, respiratorias, renales, hepáticas, gastrointestinales, infecciosas y otras) en los pacientes analizados con un RR de 0.92 (intervalo de confianza [IC] del $95 \%$ : 0.86-0.98). Sin embargo, este trabajo sumó los resultados de pacientes pediátricos junto con los de adultos, pacientes de cirugía de trauma, quemados, hipoalbuminemia, ascitis y otros como un solo grupo de pacientes críticos, como si verdaderamente fueran comparables. Cuando se analizó la mortalidad, se encontró un resultado en contra del uso de albúmina con un RR de 1.02 (IC 95 \%: 0.86-1.22) y no se encontró ninguna disminución significativa del riesgo de complicaciones cardiovasculares, respiratorias e infecciosas $^{39}$. Jiang, et al. analizaron los estudios que contrastaban la albúmina con otros líquidos para la reanimación de pacientes con sepsis y determinaron que el RR de mortalidad era de 0.94 (IC $95 \%$ : 0.87-1.02; $p=0.15$ ), es decir, sin beneficio sobre la mortalidad de los pacientes al utilizar albúmina como líquido principal o secundario durante la reanimación ${ }^{40}$. Muchos otros trabajos han demostrado beneficios en desenlaces intermedios, como menor necesidad de cristaloides a infundir, menor tiempo de vasopresores y menor tiempo de choque, pero con el mismo resultado sobre la mortalidad, por lo que no se debe recomendar el uso rutinario de albúmina como líquido de reanimación.

El resto de los coloides disponibles son sintéticos y se clasifican según sus características farmacocinéticas; por ejemplo, respecto al hidroxietilalmidón (HES) al $6 \% 450 / 0.5 / 5$, el primer valor es la concentración de la suspensión (entiéndase la capacidad de incrementar el volumen plasmático por cada litro infundido [al $6 \%$ es isooncótico con un $100 \%$ de expansión; al $10 \%$ es hiperoncótico con $>100 \%$ de expansión]). El segundo valor es el peso molecular medio (PMM) expresado en kDa (< $130 \mathrm{kDa}$ : PMM bajo; de 130 a 270 kDa: PMM medio; > 270: PMM alto); a medida que aumenta el PMM se alarga la vida media y disminuye la capacidad de depuración renal. El tercer valor es la sustitución molar que determina la capacidad de degradación del coloide por la $\alpha$-amilasa: a mayor valor, más efectos adversos. Finalmente, el cuarto número es la relación C2:C6, que señala cuántos grupos hidroxietil están en el segundo átomo de carbono (C2) en relación con cuántos están en el sexto átomo de carbono (C6); una relación mayor a favor de C2 significa menor degradación mediante la $\alpha$-amilasa y mayor poder de expansión de volumen ${ }^{41}$.

En general, la evidencia disponible sobre los «almidones» como fluidos para reanimación es desfavorable, pues, a pesar de mostrar resultados intermedios aceptables, ninguno ha demostrado tener un impacto positivo en la mortalidad; incluso algunos estudios están claramente en contra de los coloides como líquido de elección durante la reanimación hídrica en diversas enfermedades. Wiedermann, et al. compararon el HES al $6 \%$ 130/0.4 con cristaloides en una muestra de 


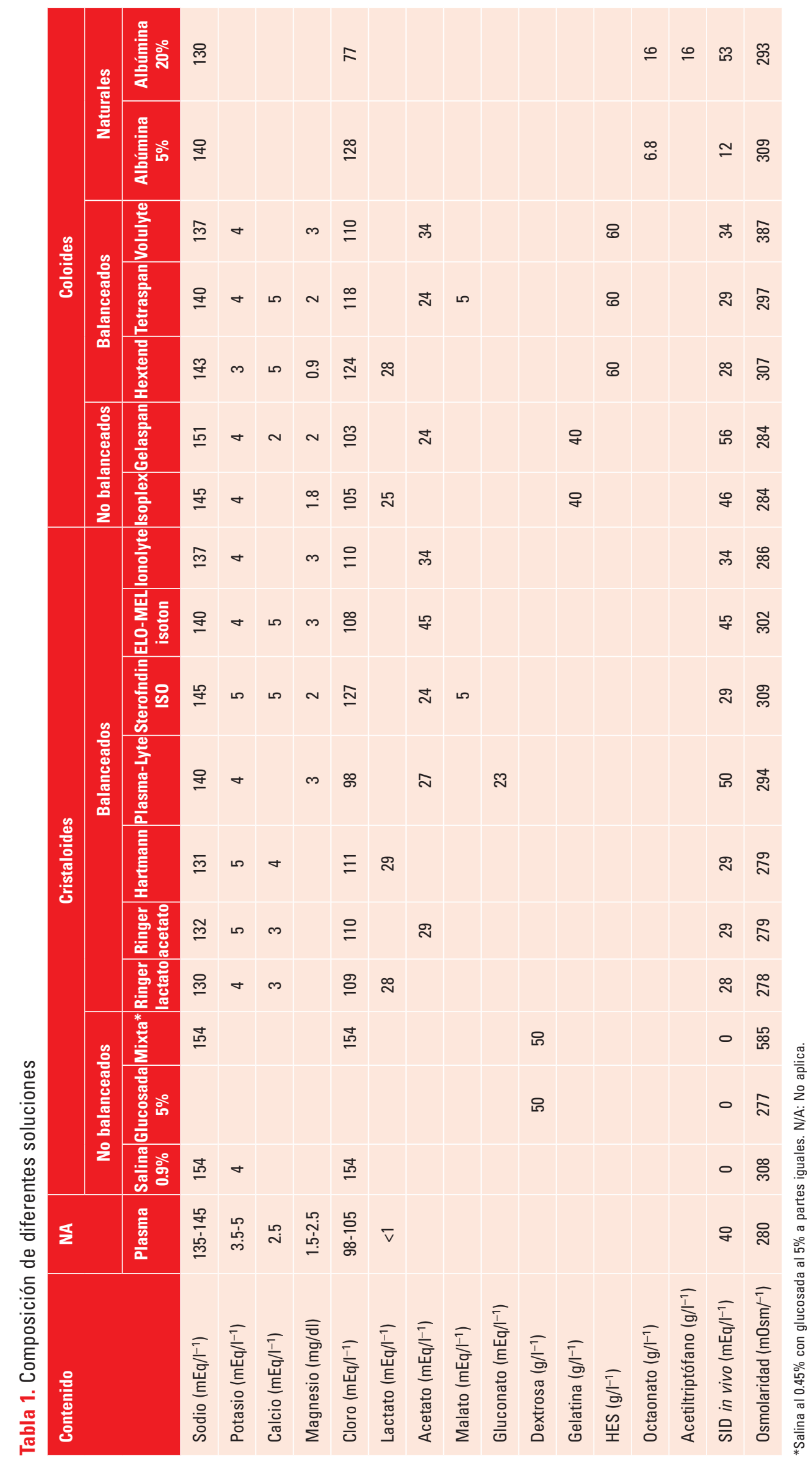


Tabla 2. Estudios que comparan soluciones no balanceadas con balanceadas

\begin{tabular}{|c|c|c|c|c|c|}
\hline $\begin{array}{l}\text { Autor, año y } \\
\text { referencia }\end{array}$ & Diseño del estudio & n & Líquidos comparados & Enfermedad & Desenlace \\
\hline Yunos, et al., $2012^{29}$ & $\begin{array}{l}\text { Observacional, } \\
\text { prospectivo, } \\
\text { abierto, antes y } \\
\text { después }\end{array}$ & 1,553 & $\begin{array}{l}\text { Clorados vs. bajos en } \\
\text { cloro }^{*}\end{array}$ & Indistinta & $\begin{array}{l}\text { Las soluciones bajas en cloro } \\
\text { disminuyen la incidencia de LRA } \\
\text { y la necesidad de TRR }\end{array}$ \\
\hline Young, et al., $2014^{30}$ & RCT, doble ciego & 65 & $\begin{array}{l}\mathrm{NaCl} 0.9 \% \text { vs. } \\
\text { Plasma-Lyte A }\end{array}$ & Trauma & $\begin{array}{l}\text { Plasma-Lyte A alcanzó mejor el } \\
\text { equilibrio ácido-base, sin } \\
\text { hipercloremia. Ambos grupos } \\
\text { presentaron la misma mortalidad }\end{array}$ \\
\hline Shaw, et al., $2014^{31}$ & $\begin{array}{l}\text { Observacional, } \\
\text { retrospectivo }\end{array}$ & 109,836 & $\begin{array}{l}\text { Ajustado por } \\
\text { concentración de cloro }\end{array}$ & Indistinta & $\begin{array}{l}\text { A mayor concentranción sérica } \\
\text { de cloro (basal y posterior a } \\
\text { reanimación), mayor mortalidad }\end{array}$ \\
\hline $\begin{array}{l}\text { Raghunathan, } \\
\text { et al., 2014 }\end{array}$ & $\begin{array}{l}\text { Observacional, } \\
\text { retrospectivo }\end{array}$ & 6,730 & $\begin{array}{l}\text { No balanceadas vs. } \\
\text { balanceadas }\end{array}$ & Sepsis & $\begin{array}{l}\text { Menor mortalidad, incidencia de } \\
\text { LRA y días de estancia en } \\
\text { pacientes con soluciones } \\
\text { balanceadas }\end{array}$ \\
\hline $\begin{array}{l}\text { Ragunathan, et al., } \\
2015^{33}\end{array}$ & $\begin{array}{l}\text { De cohorte } \\
\text { retrospectiva }\end{array}$ & 60,734 & $\begin{array}{l}\mathrm{NaCl} 0.9 \% \text { vs. } \mathrm{NaCl} \\
0.9 \% \text { con balanceadas } \\
\text { vs. } \mathrm{NaCl} 0.9 \% \text { con } \\
\text { coloides vs. } \mathrm{NaCl} 0.9 \% \\
\text { con balanceadas y } \\
\text { coloides }\end{array}$ & Sepsis & $\begin{array}{l}\text { Menor mortalidad en pacientes } \\
\text { tratados con } \mathrm{NaCl} 0.9 \% \text { y } \\
\text { balanceadas que en los otros } \\
\text { grupos. Mayor mortalidad en el } \\
\text { grupo de } \mathrm{NaCl} 0.9 \% \text { con } \\
\text { coloides (y mayor costo) }\end{array}$ \\
\hline Young, et al., $2015^{34}$ & $\begin{array}{l}\text { RCT, muticéntrico, } \\
\text { ciego, doble } \\
\text { cruzado }\end{array}$ & 2,092 & $\begin{array}{l}\mathrm{NaCl} 0.9 \% \text { vs. } \\
\text { Plasma-Lyte } 148\end{array}$ & Indistinta & $\begin{array}{l}\text { Sin diferencia en la mortalidad, } \\
\text { la incidencia de LRA o la } \\
\text { necesidad de TRR (seguimiento a } \\
90 \text { días) }\end{array}$ \\
\hline Wu, et al., $2011^{35}$ & RCT, multicéntrico & 40 & $\begin{array}{l}\mathrm{NaCl} 0.9 \% \text { vs. Ringer } \\
\text { lactato }\end{array}$ & Pancreatitis & $\begin{array}{l}\text { Disminución de SRIS y PCR en el } \\
\text { grupo de Ringer lactato }\end{array}$ \\
\hline Sethi, et al., $2017^{36}$ & Retrospectivo & 115 & $\begin{array}{l}\text { Salina } 0.9 \% \text { vs. Ringer } \\
\text { lactato } 0 \text { Isolyte }\end{array}$ & Sepsis & $\begin{array}{l}\text { Disminución de la mortalidad con } \\
\text { soluciones balanceadas }\end{array}$ \\
\hline Self, et al., $2017^{37}$ & RCT multicéntrico & 13,347 & $\begin{array}{l}\text { Salina } 0.9 \% \text { vs. } \\
\text { soluciones } \\
\text { balanceadas (Ringer } \\
\text { lactato o Plasma-Lyte) }\end{array}$ & Indistinta & $\begin{array}{l}\text { Disminución de la mortalidad, la } \\
\text { necesidad de TRRI y de LRA } \\
\text { persistente en el grupo de } \\
\text { soluciones balanceadas }\end{array}$ \\
\hline
\end{tabular}

*Salina al $0.9 \%$, gelatina al $4 \%$ o albúmina al $4 \%$ en salina frente a Hartmann, Plasma-Lyte 148 o albúmina al $20 \%$.

RCT: ensayo controlado aleatorizado. PCR: Proteína $C$ reactiva.

pacientes y encontraron que el HES incrementó la mortalidad con un RR de 1.25 (IC $95 \%$ : 0.98-1.58; $p=0.069)^{42}$. Gattas, et al. compararon los estudios de HES al $6 \% 130 / 0.4$ en pacientes mediante un metaanálisis que demostró un incremento de la mortalidad en pacientes con HES con un RR de 1.08 (IC $95 \%$ : 1.001.17), además de incrementar la necesidad de terapia de remplazo renal (TRR) con un RR 1.25 (IC $95 \%$ : 1.08-1.44) $)^{43}$. El estudio $6 \mathrm{~S}$ mostró resultados similares en pacientes con sepsis severa: aumento de la mortalidad a los 90 días con un RR de 1.17 (IC $95 \%$ : 1.01-1.36) y mayor riesgo de requerir TRR ${ }^{44}$. El estudio CRISTAL determinó que no había diferencia significativa en la mortalidad a los 28 días dentro de UCI ni durante la hospitalización, con un resultado discretamente favorable para los coloides en la mortalidad a los 90 días. En este mismo estudio, el grupo de pacientes con sepsis no presentó diferencia significativa en ninguno de los desenlaces ${ }^{45}$.

En relación con las gelatinas, Thomas-Rueddel, et al. publicaron un metaanálisis -la mayoría con pacientes de cirugía cardíaca u ortopédica- que determinó que los pacientes tratados con gelatinas intravenosas (poligelina, gelatina, oxipoligelatina y otros modificados) presentaron una mortalidad similar al control, con tendencia a favor del control, al igual que 
en relación con la necesidad de hemoderivados y la incidencia de lesión renal aguda (LRA) ${ }^{46}$. Los resultados de otros estudios son similares ${ }^{21,22}$.

\section{Hemoderivados}

Los hemoderivados son fluidos de composición mixta, con elementos disueltos y suspendidos que pueden cambiar sus propiedades dependiendo de las características del medio. A continuación se discuten los tres componentes de la sangre más utilizados durante la reanimación: concentrado eritrocitario (CE), plasma fresco congelado (PFC) y concentrado plaquetario (CP). Efectos secundarios como TRALI y TACO tienen mayor incidencia con el CE, pero se pueden presentar con todo tipo de hemoderivados y de forma independiente a la dosis; estas dos complicaciones merecen una revisión específica más profunda fuera de este manuscrito.

\section{Concentrado eritrocitario}

El CE es el hemoderivado más utilizado en reanimación, particularmente en pacientes con traumatismos. Se separa de la sangre total y contiene una cantidad mínima de plasma, mezclada con diferentes conservadores que modifican sus características ${ }^{47}$. También el tiempo y temperatura de almacenamiento causan una reducción de la actividad de la bomba sodio-potasio de las membranas eritrocitarias, lo que explica las concentraciones bajas de sodio y altas de potasio. Las alteraciones del metabolismo de los glóbulos rojos generan elevadas concentraciones de lactato ${ }^{47,48}$. Por si fuera poco, las bajas temperaturas de almacenaje ocasionan una disminución del 2,3-difosfoglicerato, que desplaza la curva de disociación de la hemoglobina hacia la izquierda. Esto hace que la hemoglobina tenga mayor afinidad por el oxígeno al pasar por los capilares pulmonares, pero libere menos oxígeno al pasar por el resto de los tejidos, efecto que dura entre 6 y $8 \mathrm{~h}$ después de la transfusión.

\section{Plasma fresco congelado}

Se trata de una solución hiperosmolar $( \pm 370 \mathrm{mOsm} / \mathrm{l})$ : concentración casi normal de albúmina, alta concentración de sodio y fosfato y baja concentración de cloro, lo que genera una SID muy alta, aproximadamente de $100 \mathrm{mEq} / \mathrm{l}$, debido a los conservadores, como el citrato trisódico (anticoagulante), el dihidrogenofosfato de sodio (amortiguador) y la dextrosa (sustrato para el metabolismo celular) ${ }^{47}$. Por estas razones, el uso de grandes cantidades de PFC puede generar alcalosis metabólica ${ }^{49}$.

\section{Concentrado plaquetario}

También se trata de hemoderivados hiperosmolares ( $\pm 350 \mathrm{mOsm} / \mathrm{l}$ ), con cantidades elevadas de sodio y bajas de cloro, con una SID aproximada de $80 \mathrm{mEq} / \mathrm{l}$. Esto modifica el estado ácido-base hacia la alcalosis metabólica ${ }^{47}$.

\section{Perspectivas}

El objetivo de la reanimación hídrica siempre se ha enfocado a la restitución de la homeostasis, la euvolemia, con restitución de las pérdidas basales 0 patológicas que presentan los pacientes. Por ello, la administración de líquidos intravenosos quizá sea la intervención terapéutica más ubicua y, a pesar de esto, existe un acúmulo creciente de información sobre efectos favorables y adversos de cada líquido utilizado en la práctica diaria. A más de 180 años del inicio del uso de soluciones intravenosas y después de cambios en los principios que se creyeron absolutos, comprendemos que para la adecuada selección de soluciones intravenosas debemos integrar la fisiología del endotelio con las características de los líquidos y la fase de reanimación en la que se encuentre el paciente, e individualizar el tratamiento en función de éstas y muchas otras variables clínicas y bioquímicas. No existe la solución intravenosa ideal como estándar para todos los casos, por lo que se requiere una mayor cantidad de estudios que comparen los cristaloides entre sí -balanceados contra no balanceados- y que nos ayuden a discriminar entre las soluciones balanceadas para adaptarlas a las diferentes enfermedades de los pacientes, siempre en su beneficio.

\section{Conflictos de intereses}

Sin conflictos de intereses que declarar.

\section{Bibliografía}

1. Rewa O, Bagshaw SM. Principles of fluid management. Crit Care Clin. 2015;31(4):785-801.

2. Janakan G, Ellis H. Dr. Thomas Aitchison Latta (c 1796-1833): pioneer of intravenous fluid replacement in the treatment of cholera. J Med Biogr. 2013;21(2):70-4

3. Myburgh JA, Mythen MG. Resuscitation fluids. N Eng J Med. 2013; 369(13):1243-51.

4. Lee AJ. Sydney Ringer (1834-1910) and Alexis Hartmann (1898-1964). Anaesthesia. 1981;36:1115-21. 
5. Guerrero M, Andrighetti G. Reposición de volumen en el politraumatizado. Rev Med Clin Condes. 2011;22(5):599-606.

6. Guarner V. Las contiendas bélicas y su aportación al conocimiento de la respuesta metabólica al trauma. Acta Médica Grupo Ángeles. 2003;1(1):53-8.

7. Foëx BA. How the cholera epidemic of 1831 resulted in a new technique for fluid resuscitation. Emerg Med J. 2003;20:316-8.

8. Raghunathan K, Shaw AD, Bagshaw SM. Fluids are drugs. Curr Opin Crit Care. 2013;19(4):290-8.

9. Woodcock TE, Woodcock TM. Revisited Starling equation and the glycocalyx model of transvascular fluid exchange: an improves paradigm for prescribing intravenous fluid therapy. Brit J Anaesthesia. 2012;108(3): 384-95.

10. Reistma S, Slaaf DW, Vink $H$, van Zandwoort MA, oude Egbrink MGA. The endotelial glycocalyx: composition, functions and visualization. Pflugers Arch. 2007; 454:345-59.

11. Becker BF, Chappell D, Bruegger D, Annecke T, Jacob M. Therapeutic strategies targeting the endotelial glycocalyx: acute déficits, but great potential. Cardiovasc Res. 2010;87:300-10.

12. Nieuwdorp $M$, van Haeften TW, Gouverneur MC, Mooij HL, van Lieshout $\mathrm{MH}$, Levi $\mathrm{M}$, et al. Loss of endotelial glycocalyx during acute hyperglycemia coincides with endotelial dysfunction and coagulation activation in vivo. Diabetes. 2006;55:480-6.

13. Nelson A, Berkestedt I, Schmidtchen A, Ljunggren L, Bodelsson M. Increased levels of glycosaminoglycans during septic shock: relation to mortality and the antibacterial actions of plasma. Shock. 2008;30:623-7.

14. Steppan J, Hofer S, Funke B, Brenner T, Hanrich M, Martin E, et al. Sepsis and major abdominal surgery lead to flaking of the endotelia glycocalix. J Surg Res. 2011:165:136-41.

15. Nieuwdrop M, Mooki HL, Kroon J, Arasever B, Spaan JA, Ince C, et al Enrothelial glycocalyx damage coicides with microalbuminuria in type 1 diabetes. Diabetes. 2006;55:1127-32.

16. Karangelis D, Asimakopoulou A, Kanakis I, Tagarakis GI, Koufakis T, Triposkiadis F, et al. Monitoring serum chondroitin sulfate levels in patients submitted to coronary artery bypass surgery. Biomed Chromatogr. 2010;25:748-50

17. Johansson PI, Stensballe J, Rasmussen LS, Ostrowski SR. A high admission syndecan-1 level, a marker of endotelial glycocalyx degradation is associated with inflammation, protein $\mathrm{C}$ depletion, fibrinolysis, and increased mortality in trauma patients. Ann Surg. 2011;254:194-200.

18. Levick JR, Michel CC. Microvascular fluid exchange and the revised Starling priciple. Cardiovasc Res. 2010;87(2):198-210.

19. Selmer MW, Tice TW. Sepsis resuscitation fluid choice and dose. Clin Ches Med. 2016;37:241-50.

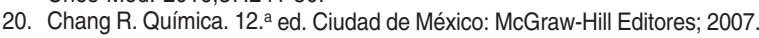

21. Bartels K, Thiele RH, Gan TJ. Rational fluid management in today's ICL practice. Critical Care. 2013;17(Suppl 1):S6. Fe de erratas en: Critical Care. 2013;17(Suppl 1):S10.

22. Garnacho-Monterjo J, Fernández-Mondéjar E, Ferrer-Roca R, Herrera-Gutiérrez ME, Lorente JA, Ruiz-Santana S, et al. Cristaloides y coloides en la reanimación del paciente crítico. Med Intensiva 2015;39(5):303-315.

23. Chowdhury AH, Cox EF, Francis ST, Lobo DN. A randomized, controlled, double-blind crossover study on the effects of $2 \mathrm{~L}$ infusions of $0.9 \% \mathrm{sa}-$ line and Plasma-Lyte 148 on renal blood flow velocity and renal cortical tissue perfusión in healthy volunteers. Ann Surg. 2012;256(1):18-24.

24. Li H, Sun S, Yap JQ, Chen J, Quian Q. $0.9 \%$ saline is neither normal nor physiological. J Zhejiang Univ Sci B. 2016;17(3):181-7.

25. Yunos NM, Bellomo R, Story D, Kellum J. Bench-to-bedside review: Chloride in critical illness. Critical Care. 2010,14(4):226.

26. Langer T, Santini A, Scotti E, van Regenmortel N, Malbrain ML, Caironi $P$. Intravenous balanced solutions: from physiology to clinical evidence. Anaesthesiol Intensive Ther. 2015;47 Spec No: s78-88.

27. Guidet B, Soni N, Della Rocca G, Kozek S, Vallet B, Annane D, et al A balanced view of balanced solutions. Crit Care. 2010:14(5):325.

28. Moritz ML, Ayus JC. Maintenance intravenous fluids in acutely ill patients. N Engl J Med. 2015;373:1350-60.

29. Yunos NM, Bellomo R, Hegarty C, Story D, Ho L, Bailey M. Association between a chloride-liberal vs. chloride-restrictive intravenous fluid administration strategy and kidney injury in critically ill adults. JAMA. 2012;308(15):1566-72.
30. Young JB, Utter GH, Schermer CR, Galante JM, Phan $\mathrm{HH}$, Yang Y, et al Saline versus Plasma-Lyte $A$ in initial resuscitation of trauma patients: a randomized trial. Ann Surg. 2014;259(2):255-62.

31. Shaw AD, Raghunathan K, Peyerl FW, Munson SH, Paluszkiewiez SM, Schermer CR. Association between intravenous chloride load during resuscitation and in-hospital mortality amog patients with SIRS. Intensive Care Med. 2014:40:1897-905.

32. Raghunathan K, Shaw A, Nathanson B, Stürmer T, Brookhart A Stefan MS, et al. Association between the choice of IV crystalloid and in-hospital mortality among critically ill adults with sepsis. Crit Care Med. 2014(7);42:1585-91.

33. Raghunathan K, Bonavia A, Nathanson $B H$, Beadles $C A$, Shaw AD, Brookhart A, et al. Association between initial fluid choice and subsequent in-hospital mortality during the resuscitation of adults with septic shock. Aneasthesiology. 2015;123(6):1385-93.

34. Young P, Bailey M, Beasley R, Henderson S, Mackle D, McArthur C et al. Effect of a buffered crystalloid solution vs. saline on acute kidney injury among patients in the intensive care unit: the SPLIT Radomized Clinical Trial. JAMA. 2015;314(16):1701-10.

35. Wu BU, Hwang JQ, Gardner TH, Repas K, Delee R, Yu S, et al. Lactated Ringer's solution reduces systemic inflammation compared with saline in patients with acute pancreatitis. Clin Gastroenterol Hepatol. 2011;9(8):710-7

36. Sethi M, Owyang CG, Meyers C, Parekh R, Shah KH, Manini AF. Choice of resuscitative fluids and mortality in emergency department patients with sepsis. Am J Emerg Med. 2018;36(4):625-9.

37. Self WH, Semler MW, Wanderer JP, Ehrenfeld JM, Byrne DW, Wang L, et al. Saline versus balanced crystalloids for intravenous fluid therapy in the emergency department: study protocol for a cluster-randomized, multiple-crossover trial. Trials. 2017;18(1):178.

38. Mitra S, Khandelwai $P$. Are all colloids same? How to select the right colloid? Indian J Anaesth. 2009;53(5):592-607

39. Vincent JL, Navickis RJ, Wilkes MM. Morbidity in hospitalized patients receiving human albumin: a meta-analysis of randomized, controlled trials. Crit Care Med. 2004;32(10):2029-38.

40. Jiang $L$, Jiang $S$, Zhang M, Zheng Z, Ma Y. Albumin versus other fluids for fluid resuscitation in patients with sepsis: a meta-analysis. PLoS ONE. 2014;9(12):e114666.

41. Agrò FE, Fires D, Benedetto M. How to maintain and restore fluid balance: colloids. En: Agrò FE, ed. Body fluid management: from physiology to therapy. 1. ${ }^{a}$ ed. Italia: Springer-Verlag; 2013. p. 47-70.

42. Wiedermann CJ, Joannidis M. Mortality after hydroxyethyl starch 130/0.4 infusion: an updated meta-analysis of randomized trials. Swiss Med Wkly. 2012;142:w13656

43. Gattas DJ, Dan A, Myburgh J, Billot L, Lo S, Finfer S, CHEST Management Committee. Fluid resuscitation with $6 \%$ hydroxyethyl starch (130/0.4) in autely ill patients: an update systematic review and meta-analysis. Anesth Analg. 2012;114:159-69.

44. Perner A, Haase N, Guttormsen AB, Tenhunen J, Klemenzson G, Åneman A, et al. Hydroxyethyl starch $130 / 0.42$ versus Ringer's acetate in severe sepsis. N Eng J Med. 2012;367(2):124-34.

45. Annane D, Siami S, Jaber S, Martin C, Elatrous S, Descorps-Declère A et al. Effects of fluid resuscitation with colloids vs crystalloids on mortality in critically ill patients presenting with hypovolemic shock - The CRISTAL randomized trial. JAMA. 2013;310(17):1809-17.

46. Thomas-Rueddel DO, Vlasakov V, Reinhart $\mathrm{K}$, Jaeschke $\mathrm{R}$, Rueddel $\mathrm{H}$ Hutagalung $R$, et al. Safety of gelatin for volume resuscitacion - a systematic review and meta-analysis. Intensive Care Med. 2012;38: 1134-42.

47. Langer T, Ferrari M, Zazzeron L, Gattinoni L, Caironi P. Effects of intravenous solutions on acid-base equilibrium: from crystalloids to coIloids and blood components. Anaesthesiol Intensive Ther. 2014;46(5): 350-60

48. Sümpelmann R, Schürholz T, Thorns E, Hausdörfer J. Acid-base, electrolyte and metabolite concentrations in packed red blood cells for major transfusions in infants. Pediatr Anaesth. 2001;11(2):169-73.

49. Raj D, Abreo K, Zibari G. Metabolic alkalosis after orthopic liver transplantation. Am J Transplant. 2003:3:1566-9. 